\title{
La Formación Docente: La Metáfora De La Caja Del Formador
}

\author{
Yolanda Márquez-Domínguez \\ Departamento de Didáctica e \\ Investigación Educativa de la Universidad de La Laguna, España \\ Josué Gutiérrez-Barroso \\ Departamento de Sociología de la Universidad de La Laguna, España \\ Ana Isabel González-Herrera \\ Departamento de Didáctica e \\ Investigación Educativa de la Universidad de La Laguna, España
}

Doi: 10.19044/esj.2018.v14n7p20 URL:http://dx.doi.org/10.19044/esj.2018.v14n7p20

\begin{abstract}
This paper focuses on the key training points needed to be a good teacher. Reflecting on the keys and elements of a good praxis often results to the assessment of training processes and the influence of these on good practices. The aim of this study titled "The Trainer's Box" is to reflect on those elements which gaive specific answers that are adapted to the different objectives, contexts, and moments which have been generalized when speaking of "The New Teaching Role", the "Ideal Teacher", or the "Effective Teacher". The starting point of this reflection is that there is not just one answer, nor a possibility of universal recommendations to the question as to what to include in the container. This, however, is in a bid to obtain an "ideal" formula on the subject of good teacher training.
\end{abstract}

Keywords: Teacher training, good practices, teacher quality

\section{Resumen}

Con este trabajo tratamos de reflexionar sobre las claves de la formación de los buenos maestros. Reflexionar sobre la buena praxis, permite la evaluación de los procesos de capacitación y la influencia de éstos en la mejora de la calidad docente. El objetivo de este ensayo; "La caja del formador", es reflexionar sobre aquellos elementos que dan respuestas específicas, adaptadas a los diferentes objetivos, contextos y momentos que se han planteado al hablar de "el nuevo rol docente", el "profesor ideal" o el "docente eficaz" ". El punto de partida de esta reflexión es que no hay una 
sola respuesta, ni recomendaciones universales a la para obtener una fórmula "ideal" sobre el tema de la buena formación docente.

Palabras-Clave: Formación docente, buenas prácticas, calidad docente

\section{Introduction}

Iniciemos esta metáfora, que procede del corazón y de la cabeza, sobre la caja del formador/a. Del corazón por lo que de pasión contiene, de la cabeza por lo que de competencias posee. Todo un compendio de objetos materiales e inmateriales que hace de este cofre un tesoro simbólico integrado de significados, principios y esencias de lo que podría ser el ideal de la formación humana. Algo etéreo, postmodernista, que resulta difícil de demostrar si no hay una evidencia científica, un hecho; pero que esconde, haciendo un símil, lo que Pascal (1623) intentó explicar sin apenas palabras que: "la grandeza de las personas inteligentes es invisible a los reyes, a los ricos, a los capitanes" (p.10). Pues así es la metáfora de la caja del formador/a, un espacio lleno de grandeza y pasión, invisible a muchos/as de los/as próceres de la Educación.

Situarnos en posición de salida, hacerse con las preguntas, entenderlas, recopilar información del entorno, del contexto, opiniones desde diferentes niveles generacionales, eso hicimos; ese fue mi primer paso en la construcción de esta alegoría sobre la formación. Primero con nuestros hijos, alumnos insertados dentro del sistema educativo de la Ley Orgánica de Mejora de la Calidad Educativa (LOMCE) de 2013; la segunda fuente de información, alguien cercano a nuestro entorno familiar que se formó, al igual que nosotros, con la Ley General de Educación (1970) y, por último, acudimos a la experiencia de años pretéritos de la educación franquista. Tres generaciones, tres puntos de vista dispuestos en torno a tres contextos educativos, sociales y políticos desiguales y que nos han ayudado a crear ese primer boceto sobre las herramientas a incluir en este cofre de formadores.

Actuando como un erudito, con su habitual buena disposición a participar en cualquier proceso investigador en el que les hemos solicitado colaboración, preguntamos a uno de nuestros hijos: "si tuvieras una caja", ¿qué objetos o palabras introducirías para definir a un/a formador/a? Dime tres cosas buenas y tres que descartarías: "No incluiría el castigo, la disciplina y el enfado. Si incluiría, querer a los niños/as y saber enseñar”. El siguiente de los interlocutores, se manifestó con una voz dubitativa, costó arrancar de él adjetivos u objetos, está claro que la experiencia no confiere inmediatez de respuesta segura y firme, al igual que lo hizo en primer lugar el niño, muy al contrario, en estas lides todos tenemos nuestras dudas al respecto de lo qué incluir o no en una formación eficiente y de calidad pero, a su vez, es curioso pensar que quizás la espontaneidad no es la mejor consejera en estas cuestiones porque si al final te implicas lo harás definiéndote, exponiéndote al juicio de 
una experiencia educativa que incluirá aquello que recuerdas con una significación positiva que ha podido marcar tú trayectoria profesional o desechar aquello que marcó una devastadora e improductiva formación educativa. Así, tras unos segundos se rompió el silencio y añadió: "incluiría: formación, transmisión e intuición y descartaría, el aburrimiento”. Por último, la voz de este coro de sabiduría, la memoria histórica de la educación tradicional en España, el testimonio donde la formación era producto de una realidad educativa hegemónica (Gramsci, 2011) gobernada por dioses que domesticaban la formación arrogándose el rol de ser el "guardián entre el centeno" (Salinger, 2006); el saber impuesto que no entiende a la persona como "ser" sino como "deber ser" y dentro de esta, un ejemplo que nos trasladó uno de sus "supervivientes" sobre su concepción, una vez integrados y eliminados los elementos con los que construiría su rompecabezas, de la formación: "dentro de la caja: disposición natural para enseñar, conciencia crítica y curiosidad. Fuera de la caja: vida privada, consejos no pedidos".

Así que, en esta reflexión sobre la caja del formador/a, hemos descubierto muchas personas que han opinado de forma convincente sobre un aspecto u otro de la pasión por la formación. Si, pasión porque "cada vez que haga algo notable iré construyendo una rampa hasta alcanzar el cielo". Reflexión de un niño, con su madura y floreciente sabiduría, lanzó, como un proyectil, en un día en los que nuestra autoestima estaba resentida, resolviendo sin paños calientes, en cuestión de segundos, nuestra frágil resiliencia; ayudando a darnos cuenta de que en realidad estas palabras son producto, quizás, de una disposición natural para enseñar, de la propia intuición y del amor que le trasladamos a nuestros alumnos y que nos llevan a cuestionar si, ¿es ésta una utopía en la formación? Integrar estos elementos son, ¿descabellados o ficción educativa? ¿Podrían ser los componentes de esta caja? De hecho, reúnen componentes que otras personas consideran importantes, entre ellas, a las que se les solicitó opinión, entonces, nuestro enigma de la formación, en parte, puede estar resuelto. Algunos de los ingredientes sobre ¿qué formador/a llegar a ser? puede estar resuelto. Por fin, algo de lo que partir, algo que nos define, encontramos la Caja, aquella metáfora, aquel símbolo que hará las veces de estandarte en nuestro caminar por la identidad profesional de la formación.

\section{- ¿Qué Caja, qué símbolo?}

Decía Latour (2017) respecto a los objetos y su mediación simbólica en las relaciones de lo humano y lo no humano, que:

(...) tomen en consideración cosas y hallarán humanos.

Tomen en consideración humanos e inmediatamente se toparán con cosas. Presten atención a cosas duras: irán suavizándose, ablandándose o humanizándose. Presten 
atención a humanos: verán cómo se vuelven eléctricos, automáticos o informáticos. Ni siquiera sabemos definir con claridad lo que a unos hace humanos y a otros técnicos, pero podemos documentar con precisión sus modificaciones y sustituciones, sus delegaciones y representaciones. (p58).

Y así es como con objetos simbólicos damos significado a nuestra identidad, lo que nos posiciona en nuestro discurrir humano, en nuestras decisiones y actuaciones. La caja como símbolo puede estar ocupada por nuestras bondades o nuestras ruindades, puede estar llena de virtudes o de vileza, puede estar habitada por nuestro yo más casto o nuestro yo indecente, o puede consagrarse a nuestra eticidad o a nuestra inmoralidad, a nuestro ego o a nuestra humildad. Carpe diem, así reza nuestra caja y así, nuestra visión de la formación como un proceso que resurge de las profundidades donde habita el pez abisal, para descubrir el futuro cuya primera sílaba ya supone un recuerdo. CARPE DIEM: VIVE EL MOMENTO.

Una vez elegido el recipiente se hace necesario ir concretando, a través de la deducción, aquello por lo que estamos aquí. Es decir, cada vez se va vislumbrando más nuestro objeto de reflexión: la caja del formador/a, ¿qué perfil docente? ¿Con qué formación? ¿Qué elementos conformarían el papel del formador/a? ¿Aquellos que den respuestas específicas, ajustadas a los diferentes objetivos, sujetos, contextos y momentos o se tratarán de elementos que se unen a la "lógica de los listados" que se han generalizado en torno al "nuevo papel docente", el "docente ideal" o el "docente efectivo"? (Torres de Castillo, 1998).

En nuestro caso, el listado no procede de un modelo adoptado acríticamente, que incorpora conceptos y marcos teóricos ideológicos diferentes y hasta contradictorios entre sí. Este modelo parte de objetos con vida propia, independientes, críticos, justos, equitativos que suponen un elemento transformador, emancipador, en definitiva, ingredientes de la formación para construir conocimiento como un acto político pasando de ser seres sociales a seres activos, críticos y pensantes. Definición que quizás desafía las habituales categorías de análisis sobre la formación o de lo que debería ser la formación del futuro en consonancia con los modelos de cunaesa cuna biológica, racial, familiar, cultural, nacional, de clase social, etc.- que predestina a ser según la jerarquía de oportunidades establecida por otros (Savater, 1997). Esta supuesta imprecisión terminológica, llena de retórica pedagógica: emancipadora, política, crítica, equitativa, no está sacada de la irreflexión sino de una realidad en permanente disputa de eso que, vagamente, se avizora hoy como la formación deseada, la "nueva" formación, "la formación del siglo XXI", "la formación vestida con piel de cordero" la del 
acomodo programado a ella... para comerte mejor, según dijo el lobo pedagógicamente disfrazado de abuelita (Savater, 1997, p.153).

En definitiva, los elementos que conforman nuestro ideal de formación no son abstracciones que supongan el desafío de construir "certezas situadas". Nuestros objetos nos definen y por tanto asignan valor a un modelo de formador/a que, a su vez, está en relación con el tipo de sociedad a la que se aspira; se trata de una fórmula sencilla: adquirir para hacer, adquirir para llegar a ser. La formación como proceso de conquista de la libertad.

\section{- $\quad$ Elementos de la caja}

- La creatividad, el arte.

La creatividad es un bien social, una decisión y un reto de futuro. Por ello, formar en creatividad es apostar por un futuro de progreso, de justicia, de tolerancia y de convivencia. Creatividad es hacer algo nuevo para bien de los demás. (Saturnino de la Torre, 2006, p. 137)

Somos más partidarios del formador/a artista, que es sobre todo cabeza, que del formador/a burócrata, que se le organiza el trabajo para que sea sólo mano ejecutora (Collet-Sabé, 2011). Así que para nosotros el primero de los elementos de esta metáfora de la formación como arte sería, siguiendo a Collet-Sabé (2011):

(...) hacer es pensar y pensar es hacer y que para formar como oficio, como arte no puede ser mediante un código cerrado, restringido (Bernstein, 1979), como un modelo catedral. Por el contrario, tiene que haber un sitio destacado para la ambigüedad, para la apertura, para el bazar, que es el peor enemigo de la burocracia, (p. 39).

\section{- La pasión, la ternura}

El diccionario define la pasión como "un sentimiento muy intenso". Es motor, fuerza, que emana de la emoción. Las personas se apasionan por cosas, asuntos, causas, etc. El apasionamiento genera energía, determinación, convicción, compromiso e, incluso, obsesión. La pasión puede llevar a desear alcanzar una meta con una visión profunda. La pasión no es lujo, no es una floritura o una cualidad excéntrica que sólo posean unos/as pocos formadores/as. La pasión es esencial para una buena enseñanza.

\section{- La educación para la ciudadanía}

Por primera vez, el hombre ha comprendido realmente que es un habitante del planeta, y tal vez piensa y actúa de una nueva manera, no sólo como individuo, familia o género, Estado o 
grupo de Estados, sino también como planetario. Vernadsky (1863).

El propósito de la formación ciudadana no sólo supondrá mejorar la convivencia, sino que orientará a la implementación de la educación integral de los/as jóvenes por la necesidad de hacer frente a esos complejos problemas sociales requeridos de una inmediata respuesta política. Tal formación les ayudará a posicionarse frente a una idea, les ayudará a plantearse metas y a rechazar imposiciones (MolinaFernández, Medero, Pérez-Jorge, \& Oda-Ángel, 2017). En definitiva, a desarrollarse como una persona con conciencia crítica y autónoma, desde la libertad que le puede dar el saber.

\section{- Objetos o valores a descartar de la caja}

La maldad, las intrigas, la incertidumbre, la ira, el egocentrismo.

Los dioses nos dan muchas sorpresas: lo esperado no se cumple y para lo inesperado un dios abre la puerta Eurípides.

A través de dos de mis libros más estimados quería plasmar como la ficción es producto de la realidad, en este caso de una realidad que ahonda, en Placeres crueles (Tolstoi, 1902) y los sentimientos más descarnados que hacen del ser humano un ser hiriente y despiadado. Shakespeare al igual que Tolstoi convierten a los/as protagonistas de sus obras en símbolos de las pasiones humanas. Ciertamente en personajes que pueden ejercer el bien y el mal. Otelo se presenta como la tragedia de la incomprensión, la ira, el poder y la manipulación; Macbeth como la ambición desmedida y Hamlet como la venganza y la locura. Los/as portadores de estos aspectos despóticos constituyen una desviación potencial para la formación y el aprendizaje. Ellos/as eliminan y aniquilan la creatividad, el arte, la pasión, la motivación por hacer. Se convierten en focos de desviación que maman del egocentrismo que cultiva la autoglorificación y la tendencia a adjudicar a los demás, extraños o no, la causa de todos los males.

En conclusión, esta caja no dará hospedaje al cruel juego del castigo, la ira o el statu quo, que nos conduce a percibir, de manera peyorativa, las palabras o actos de los demás, a seleccionar lo que es desfavorable, a eliminar lo que es favorable, a seleccionar nuestros recuerdos gratificantes, a eliminar o transformar los deshonrosos. Esta caja, contendrá el amor por aprender, por formarse, desde un planteamiento humanista, filosófico, socrático. Porque, en el mejor de los casos, la formación, la enseñanza, el aprendizaje, será una vocación basada en el amor como valor fundamental de la formación y de los formadores (Pérez Jorge, 1999; Pérez-Jorge, 2010, 2010b). 


\section{- $\quad$ El enigma de la formación}

Unos, centrados en el yo de los formadores/as; otros, en su trabajo; algunos, en la importancia del compromiso, la satisfacción en el trabajo, la identidad y la influencia de la normativa sobre ellos/as. Otros opinan sobre la compresión emocional y los fines morales de los docentes y la importancia de la pasión colectiva en las escuelas, en cuanto a comunidades de aprendizaje, al igual que el ámbito no formal. En la mayoría de las investigaciones y escritos, en la práctica, todos/as interactúan y constituyen el todo.

Pero para ello hay que partir de la idea de que no hay respuesta única, ni posibilidad de recomendaciones universales, para la pregunta acerca de qué incluir en el recipiente para obtener una fórmula "ideal" sobre la cuestión de la formación, de manera específica. No la hay por varias razones: la gran diversidad de contextos que hace difícil la generalización, así como los diversos significados que adopta o puede adoptar, en cada caso, la formación (Pérez-Jorge y Rodríguez, 2012).

Por ello se hacen necesarias una serie de apreciaciones sobre el concepto de formación, las cuáles nos ayudarán a encajar, con la precisión de un cirujano. Hay que partir diciendo que la elección de este término, formación, frente al de educación, enseñanza o entrenamiento -no hablemos de adoctrinamiento- no es casual, nos puede ofrecer algunas pistas iniciales respecto al ámbito conceptual que pretendemos describir. formación.

Menze (1980) distingue tres tendencias contrapuestas del concepto de

- La primera postura entiende que es imposible entender la formación, dentro de la educación, como un concepto técnico por la esencia filosófica que los sustenta, así lo entiende Menze (1980) cuando justifica que:

(...) por razón de su origen histórico y de las implicaciones metafísicas que le son propias, está tan viciado y tiene tantas significaciones, que no sólo es imposible hacer un uso razonable de él, sino que, además, quien lo emplea se expone a la sospecha de ideología" (p.267).

Los/as partidarias de esta postura argumentan que la formación, incluye dimensiones que no pueden ser investigadas, y por tanto rechazan su empleo. No es ésta, evidentemente, la postura que nos define.

- Una segunda tendencia que identifica Menze (1980) es la de recurrir al concepto formación para identificar múltiples y a veces contradictorios conceptos.

En esta línea se plantea la formación como polisémica en cuanto a la diversidad de dimensiones, que no se limita, exclusivamente a un solo campo profesional, sino que se refiere a múltiples dimensiones: formación del 
consumidor, formación de los padres, formación sexual. En este sentido, Ferry (1991) plantea que:

(...) los significados que actualmente se están dando al concepto formación están desvirtuando el concepto (...) la noción de formación se ha cargado de tantos equívocos y aparece de tal manera pervertida por la utilización que se hace de ella, desde la extensión de la educación permanente, convertida en una empresa distribuidora de saberes etiquetados y de pequeñas seguridades, que por esto propone abandonar definitivamente el término formación" (p.52).

No compartimos, tampoco esta apreciación. Parece, y ésta es la tercera tendencia identificada por Menze (1980), y con la que más nos identificamos, que en la actualidad no tiene sentido eliminar el concepto formación, puesto que: "Formación no es ni un concepto general que abarque la educación y la enseñanza, ni tampoco subordinado a éstos" (p.269). La educación, para este autor, en palabras de Marcelo (1995), es: "una acción que se lleva a cabo desde fuera para contribuir al desarrollo personal y social de los individuos, y generalmente referida a sujetos no adultos" (p.4). En este sentido, cuando se relaciona el concepto de "Formación" con las acciones llevadas a cabo con adultos, esto significa que van dirigidas a la adquisición de saberes y de saber hacer más que saber ser, es más formal en cuanto a su organización. Por tanto, el concepto de formación siempre se asocia con alguna actividad, es decir, "formación para algo" (Honoré, 1980). Y entendida así, como función social, de transmisión de saberes, de saber hacer o de saber ser, se le supone como un beneficio para la producción, para el sistema, o cultura dominante.

La formación también puede entenderse como proceso de desarrollo personal interno y experiencial: "el proceso de desarrollo que sigue el sujeto humano hasta alcanzar un estado de 'plenitud' personal" (Zabalza, 1990a p.201); o bien, también se puede hablar de formación como "formación en el trabajo", refiriéndose a la estructura organizativa que planifica y desarrolla las actividades de formación; o también, desde el punto de vista de Ferry (1991), Pérez-Jorge, Leal y Rodríguez (2012); formarse no puede ser más que un trabajo sobre sí mismo, libremente imaginado, deseado y perseguido, realizado a través de medios que se ofrecen o que uno mismo se procura.

Así la formación tiene diferentes aristas desde las cuáles puede ofrecer formación para organizar al sujeto desde fuera o desde aquella otra que parta desde el mismo sujeto, es decir, aquella que se activa como iniciativa personal. Así, se interpreta que existe un componente, dentro del concepto de formación, axiológico, que va dirigido a alcanzar unas metas relacionadas con el desarrollo personal frente a lo meramente instrumental o técnico. La formación en este sentido engloba elementos que entran a formar parte del entorno ideológico, cultural que ayudan a sumar experiencia gracias a la interacción 
del sujeto con el medio. Es decir, se trataría de la formación como proceso (Pérez-Jorge, Alegre, Rodríguez-Jiménez, Márquez-Domínguez, \& De la Rosa-Hormiga (2016).

Pero este componente personal de la formación no significa que se realice de forma autónoma. En este sentido Debesse (1982) distingue tres procesos:

- $\quad$ Autoformación. El individuo participa de manera independiente y es el que organiza su proceso y resultados de formación. Planifica la formación.

- Heteroformación. La formación se organiza desde fuera por especialistas.

- $\quad$ Y, por último, la interformación, que el autor define como: "la acción educativa que se ejerce entre los futuros enseñantes o entre maestros y que haya un soporte de excepción en el trabajo del 'equipo pedagógico', tal como hoy se lo concibe para la formación del mañana" (Debesse, 1982, p.30).

Esta concepción de la formación conecta con la tradición de pensamiento, alemán que utiliza el término "Bildung", para referirse a la formación. "Bildung" significa tanto formación como configuración de la educación de un sujeto autoconsciente (Vierhaus, 2002) en el sentido de un proceso de crecimiento y desarrollo personal o cultural. Gadamer (1900), por su parte, explica al respecto que "el resultado de Bildung no se consigue en la forma de una construcción técnica. Bildung como tal no puede ser una meta, no puede ser vista, sino en la temática reflexiva del educador. El concepto de Bildung trasciende el de meramente cultivar talentos (citado por Beyer et al. 1989, p.80).

Pero la formación no sólo ha de entenderse como, únicamente un proceso de formación auto-formativo en su sentido más filosófico. Dentro de la conceptualización de la formación han aparecido otras visiones que han querido desarrollar una teoría de la formación para imprimirle carácter. Más concretamente, Honoré (1980) propone utilizar el término Formática para referirse al campo de conocimiento que estudie los problemas relativos a la formación y por su parte, Menze (1980) reclama que:

La teoría de la formación tiene el quehacer de poner en claro los presupuestos antropológicos del hecho de la formación, fijar el camino que tiene que recorrer el hombre en el proceso de hacerse 'hombre', e indicar de qué manera se le puede ayudar en este empeño mediante un influjo metódico, con arreglo a un plan (p.270).

Dentro de la teoría de la formación, se ha capturado otro concepto que la hace distinguirse, nos referimos al concepto de "acción formativa" o "acciones de formación". Con este concepto, vemos como la formación no sólo se dirige a esa formación de proceso de transmisión de saber, de saber hacer o llegar a ser; la acción formativa pone en conexión un conjunto de 
conductas, de interacciones entre un/a formador/a y los/as formados/as, que persiguen distintas finalidades y deseos de formación, y por las cuales se persiguen o existe intencionalidad de cambio (Pérez-Jorge, Barragán-Medero, \& Molina-Fernández, 2017). Por este hecho, el cambio se ha percibido como el gran elemento que le supone problemas a la formación, ¿Por qué? Porque, ¿cómo se puede descubrir que existe cambio en aquellas personas que ya están formadas? Está claro que las acciones formativas al desarrollarse en un contexto determinado, llámese empresa, organización, institución, etc., se desarrollan bajo una cultura empresarial, bajo unas reglas de funcionamiento y dentro de esta estructura el/la formador/a junto con los/as futuros formados/as eligen medios, métodos, objetivos específicos, evaluación para alcanzar, a través de la intervención y participación del formado/a, un cambio consciente que coadyuve a conseguir los objetivos explicitados.

Así pues, el enigma de la formación es un hecho que interpretamos como un fenómeno complejo y diverso, enigmático, en torno al cual existen demasiadas conceptualizaciones y menos acuerdos respecto a las dimensiones y teorías más relevantes para su análisis (Márquez-Domínguez, 2016). Pero a pesar de suponerle un enigma, hemos de concluir, que:

- $\quad$ En primer lugar, la formación, ha de entenderse como una realidad conceptual que no se identifica ni diluye dentro de otros conceptos al uso como educación o enseñanza.

- $\quad$ En segundo lugar, el concepto formación incorpora una dimensión personal, de desarrollo humano que es preciso tomar en cuenta frente a otras concepciones eminentemente técnicas.

- $\quad$ En tercer lugar, el concepto formación tiene que ver con la capacidad de formación, así como con la voluntad de formación. Es decir, es el individuo, la persona el responsable último de la activación y desarrollo de procesos formativos para favorecer el cambio.

\section{References:}

1. BOE núm. 187, de 6 de agosto de 1970, páginas 12525 a 12546. Ley 14/1970, de 4 de agosto, General de Educación y Financiamiento de la Reforma Educativa.

2. BOE núm. 295, de 10 de diciembre de 2013, páginas 97858-97921. Ley Orgánica 8/2013, de 9 de diciembre, para la mejora de la calidad educativa.

3. Beyer, L. et al. (1989). Preparing teachers as professionals, Chicago, Teacher College Press.

4. Collet-Sabé, J. (2011). Educació:¿ art, burocracia o artesania? Per una nova metàfora de la teoria $\mathrm{i}$ de la pràctica educativa. Pedagogia $i$ Treball Social, 1(1), 27-50. 
5. Debesse (1982). Un problema clave de la educación escolar contemporánea. En M. Debesse y G. Mialaret (Eds.). La formación de los enseñantes, (pp. 13-34). Barcelona, España: Oikos-Tau.

6. De la Torre, S. (2006). Comprender y evaluar la creatividad. Barcelona, España: Aljibe.

7. Fernández Galán, R. (2017). La ternura. Madrid, España: Alfaguara.

8. Ferry, G. (1991). El trayecto de la formación. Madrid, España: Paidós.

9. Freire, P. (1996). Política y Educación. México: Siglo XXI.

10. Fried, R. (1995). The passionate teacher: a practical guide. Boston, USA: Beacon Press.

11. Gadamer, H. (1991). Verdad y método. Salamanca, España: Sígueme.

12. Gramsci, C. (1991). Odio a los indiferentes. Madrid, España: Ariel.

13. Honoré, B. (1980). Para una teoría de la formación. Madrid, España: Narcea.

14. Latour, B. (2017). Lecciones de sociología de las ciencias, Barcelona, España: Arpa Editores.

15. Marcelo, C. (1995). Formación del profesorado para el cambio educativo. Barcelona, España: EUB.

16. Márquez Domínguez, Y. (2016). Formación de la conciencia crítica y valores ciudadanos desde el punto de vista del profesorado (tesis doctoral). Universidad de La Laguna, , Canarias, España.

17. Menze, C. (1981). Formación. En J. Speck y otros (Eds.). Conceptos fundamentales de Pedagogía, (pp. 267-297). Barcelona, Herder.

18. Pascal, B. (1993). Pensamientos. Barcelona: Altaya.

19. Molina-Fernández, E., Medero, F. B., Pérez-Jorge, D., \& Oda-Ángel, F. (2017). Cultural Empowerment and Language: Teaching Spanish to the Socially Disadvantaged Amazigh Population through the Alehop Programme. Asian Social Science, 13(10), 43-54.

20. Pérez Jorge, D. (1999). Reforma versus innovación. Educadores: Revista de la Federación Española de Religiosos de Enseñanza, 41(192), 443-455.

21. Pérez-Jorge, D. (2010). Actitudes y concepto de la diversidad humana: Un estudio comparativo en centros educativos de la isla de Tenerife (tesis doctoral). Universidad de La Laguna, Canarias, España.

22. Pérez-Jorge, D. (2010b). El profesor motivador: El profesor que estimula e implica a los alumnos en proyectos para el desarrollo de la competencia cultural y artística. En O. Alegre, O. (Ed.), Capacidades docentes para atender a la diversidad (pp.139-153). Sevilla, España: MAD.

23. Pérez-Jorge, D. \& Rodríguez, M. C. (2012). Actitudes de los maestros hacia las necesidades especiales de los alumnos. En O. Alegre, (Ed.), 
Investigación sobre competencias y tecnología para la inclusión y la interculturalidad (pp.180-196). Madrid, España: Arial.

24. Pérez-Jorge, D., Leal, E., \& Rodríguez, M.C. (2012). Voces de los maestros de Educación Especial en un centro Inclusivo: Redefinición de responsabilidades. En O. Alegre, (Ed.), Investigación sobre competencias y tecnología para la inclusión y la interculturalidad (pp.135-146). Madrid, España: Arial.

25. Pérez-Jorge, D., Alegre, O., Rodríguez-Jiménez, M., MárquezDomínguez, Y. \& De la Rosa-Hormiga, M. (2016). La Identificación Del Conocimiento Y Actitudes Del Profesorado Hacia Inclusión De Los Alumnos Con Necesidades Educativas Especiales. European Scientific Journal, http://dx.doi.org/10.19044/esj.2016.v12n7p64 12(7), 64-81.

26. Pérez-Jorge, D., Barragán-Medero, F. \& Molina-Fernández, E. (2017). A Study of Educational Programmes that Promote Attitude Change and Values Education in Spain, Asian Social Science, 13(7), 112-130.

27. Salinger, J. (1978). El Guardián entre el centeno. Madrid, España: Alianza Editorial.

28. Savater, F. (1997). El valor de educar. Barcelona: Ariel.

29. Torres del Castillo, R. (1998). Nuevo papel docente. ¿Qué modelo de formación y para qué modelo educativo? Perfiles educativos, 82. Recovered from: http://www.redalyc.org/html/132/13208202/

30. Vernadsky, V. (1997). The biosphere. New York, USA: Copernicus.

31. Vierhaus, R. (2002). Formación (Bildung). Sepatata Revista de educación y pedagogía, 14(33), 5-68.

32. Zabalza Beraza, M. (1987). Los diarios de los profesores como documentos para estudiar cualitativamente los dilemas prácticos de los profesores, (proyecto de Investigación de Acceso a Cátedra). Santiago, España. 